\title{
Blow-up for Semidiscretization of Semilinear Parabolic Equation With Nonlinear Boundary Condition
}

\author{
Ardjouma Ganon ${ }^{1}$, Manin Mathurin Taha ${ }^{1} \&$ Kidjégbo Augustin Touré ${ }^{1}$ \\ ${ }^{1}$ Institut National Polytechnique Houphouët-Boigny de Yamoussoukro, Côte d'Ivoire \\ Correspondence: Ardjouma Ganon, Unité Mixte de Recherche et d'Innovation Mathématiques et Nouvelles Technologies \\ de l'Information, Institut National Polytechnique Houphouët-Boigny de Yamoussoukro, Côte d'Ivoire. \\ E-mail: ardjganon@gmail.com
}

Received: July 23, 2019 Accepted: August 13, 2019 Online Published: September 9, 2019

doi:10.5539/jmr.v11n5p1 URL: https://doi.org/10.5539/jmr.v11n5p1

\begin{abstract}
This paper deals with the study of the numerical approximation for the following semilinear equation with a nonlinear absorption term $u_{t}=u_{x x}-\lambda u^{p}, 0<x<1, t>0$, and a nonlinear flux boundary condition $u_{x}(0, t)=0, u_{x}(1, t)=$ $u^{q}(1, t), t>0$. We give conditions under which the positive semidiscrete solution blows up in a finite time. Convergence of the numerical blow-up time to the theoretical one when the mesh size goes to zero is established. Finally, we use an efficient algorithm to estimate the blow-up time.
\end{abstract}

Keywords: semilinear equation, numerical blow-up, nonlinear boundary, finite differences, arc length transformation, Aitken $\Delta^{2}$ method

\section{Introduction}

Consider the following semilinear parabolic problem

$$
\left\{\begin{array}{l}
u_{t}=u_{x x}-\lambda u^{p}, \quad 0<x<1, t>0 \\
u_{x}(0, t)=0, u_{x}(1, t)=u^{q}(1, t), \quad t>0 \\
u(x, 0)=u_{0}(x)>0, \quad 0 \leq x \leq 1,
\end{array}\right.
$$

where $p, q>1, \lambda>0$ are given constants, and $u_{0}$ is a positive smooth function defined on $[0,1]$ such that $u_{0}^{\prime}(0)=0$ and $u_{0}^{\prime}(1)=u_{0}^{q}(1)$. It is proved that regularity solutions exist for this problem see (Gómez, Márquez, \& Wolanski, 1993). For differential equations, solutions can become unbounded in finite time, we say that they blow up, or they can be defined for all time and we call them global solutions. We call a blow-up point, the point of the space where the solution become unbounded in finite time.

For problem (1), Gómez J. L. et al. (Gómez et al., 1993) prove that under certain conditions on $u_{0}, \lambda, p$ and $q$, blow-up occurs in finite time at the boundary $x=1$; in particular,

- when $p<q$ and $u_{0}>u_{\lambda}$ where $u_{\lambda}$ is the unique positive stationary solution,

- when $p=q, u_{0}>0$ and $\lambda<1$.

Rossi J. D. (Rossi, 1998) investigated the blow-up rate for positive solutions of problem (1). He also characterize the blow-up profile in similarity variables. Problem (1) can be considered as a heat conduction problem. In this case, $u$ represents the temperature, see (Assalé, Boni, \& Diabate, 2008).

we focus in this paper on the numerical approximations of (1). Since the solution $u$ blows up in finite time, it is worth asking what can be stated about numerical approximations of this type of problems. For previous work on numerical approximations of blowing up solutions we refer to (Abia, López-Marcos, \& Martínez, 1996; Adou, Touré, \& Coulibaly, 2019; Assalé et al., 2008; Dratman, 2010; Edja, Touré, \& Koua, 2018; Ganon, Taha, \& Touré, 2019; N'dri, Touré, \& Yoro, 2018; Touré, N'Guessan, \& Diabate, 2015; Taha, Touré, \& Mensah, 2012 and references therein).

This paper is structured as follows : in section 2, we introduce a semidiscrete scheme of the problem (1). In Section 3 , we give some properties of this semidiscrete scheme. In Section 4, under suitable conditions, we show that the semidiscrete solution blows up in a finite time and this numerical blow-up time converges to the theoretical one when the mesh size goes to zero. Finally, in section 5, we illustrate our analysis by giving some numerical results. 


\section{Semidiscrete Problem}

Let $I$ be a positive integer and define the grid $x_{i}=i h, i=0, \ldots, I$, where $h=\frac{1}{I}$ is the mesh parameter. We approximate the solution $u$ of the problem (1) by the solution $U_{h}(t)=\left(U_{0}(t), \ldots, U_{I}(t)\right)^{T}$ of the following semidiscrete scheme

$$
\begin{aligned}
\frac{d U_{i}(t)}{d t} & =\delta^{2} U_{i}(t)-\lambda U_{i}^{p}(t), \quad i=0, \ldots, I-1, \quad t \in\left(0, T_{h}\right), \\
\frac{d U_{I}(t)}{d t} & =\delta^{2} U_{I}(t)+\frac{2}{h} U_{I}^{q}(t)-\lambda U_{I}^{p}(t), \quad t \in\left(0, T_{h}\right), \\
U_{i}(0) & =\varphi_{i}>0, \quad i=0, \ldots, I,
\end{aligned}
$$

where for $t \in\left(0, T_{h}\right)$,

$$
\begin{aligned}
\delta^{2} U_{i}(t) & =\frac{U_{i+1}(t)-2 U_{i}(t)+U_{i-1}(t)}{h^{2}}, \quad i=1, \ldots, I-1, \\
\delta^{2} U_{0}(t) & =\frac{2 U_{1}(t)-2 U_{0}(t)}{h^{2}}, \\
\delta^{2} U_{I}(t) & =\frac{2 U_{I-1}(t)-2 U_{I}(t)}{h^{2}}
\end{aligned}
$$

and $\left[0, T_{h}\right)$, the maximal time interval on which $\left\|U_{h}(t)\right\|_{\infty}=\max _{0 \leq i \leq I}\left|U_{i}(t)\right|$ is finite. We say that $U_{h}(t)$ blows up in a finite time if the time $T_{h}$ is finite, and $T_{h}$ is called the blow-up time of $U_{h}(t)$.

Denote

$$
\delta_{*}^{2} U_{i}(t)=\left\{\begin{array}{l}
\delta^{2} U_{i}(t) \quad \text { if } i=0, \ldots, I-1, \\
\delta^{2} U_{I}(t)+\frac{2}{h} U_{I}^{q}(t) \quad \text { if } i=I .
\end{array}\right.
$$

\section{Properties of the Semidiscrete Problem}

The below comparison lemma is another form of the maximum principle for the semidiscrete equations.

Lemma 1 Let $f \in C^{0}(\mathbb{R}, \mathbb{R})$, if $V_{h}, W_{h} \in C^{1}\left([0, T), \mathbb{R}^{I+1}\right)$ are such that

$$
\begin{aligned}
\frac{d V_{i}(t)}{d t}-\delta^{2} V_{i}(t)-f\left(V_{i}(t)\right) & <\frac{d W_{i}(t)}{d t}-\delta^{2} W_{i}(t)-f\left(W_{i}(t)\right), \quad i=0, \ldots, I, \quad t \in(0, T), \\
V_{i}(0) & <W_{i}(0), \quad i=0, \ldots, I,
\end{aligned}
$$

then we have $V_{i}(t)<W_{i}(t), \quad 0 \leq i \leq I, \quad t \in(0, T)$.

Proof. Let us define the functions $Z_{i}(t)=W_{i}(t)-V_{i}(t), 0 \leq i \leq I, t \in[0, T)$. Let $t_{0}$ be the first $t \in(0, T)$ such that $Z_{i}(t)>0$ for $t \in\left[0, t_{0}\right), 0 \leq i \leq I$, but $Z_{i_{0}}\left(t_{0}\right)=0$ for a certain $i_{0} \in\{0, \ldots, I\}$. It is not difficult to see that

$$
\begin{aligned}
& \frac{d}{d t} Z_{i_{0}}\left(t_{0}\right)=\lim _{k \rightarrow 0} \frac{Z_{i_{0}}\left(t_{0}\right)-Z_{i_{0}}\left(t_{0}-k\right)}{k} \leq 0, \\
& \delta^{2} Z_{i_{0}}\left(t_{0}\right)=\frac{Z_{i_{0}+1}\left(t_{0}\right)-2 Z_{i_{0}}\left(t_{0}\right)+Z_{i_{0}-1}\left(t_{0}\right)}{h^{2}} \geq 0 \quad \text { if } 1 \leq i_{0} \leq I-1, \\
& \delta^{2} Z_{i_{0}}\left(t_{0}\right)=\frac{2 Z_{1}\left(t_{0}\right)-2 Z_{0}\left(t_{0}\right)}{h^{2}} \geq 0 \quad \text { if } i_{0}=0, \\
& \delta^{2} Z_{i_{0}}\left(t_{0}\right)=\frac{2 Z_{I-1}\left(t_{0}\right)-2 Z_{I}\left(t_{0}\right)}{h^{2}} \geq 0 \quad \text { if } i_{0}=I,
\end{aligned}
$$

which implies that

$$
\frac{d}{d t} Z_{i_{0}}\left(t_{0}\right)-\delta^{2} Z_{i_{0}}\left(t_{0}\right)-f\left(W_{i_{0}}\left(t_{0}\right)\right)+f\left(V_{i_{0}}\left(t_{0}\right)\right) \leq 0,
$$

but this inequality contradicts (5) and the proof is complete.

Lemma 2 Let $U_{h}$ be a solution of (2)-(4). Then,

$$
U_{i}(t)>0, \quad i=0, \ldots, I, t \in\left[0, T_{h}\right) .
$$


Proof. Let $t_{0}$ be the first $t \in\left(0, T_{h}\right)$ such that $U_{i}(t)>0$ for $t \in\left[0, t_{0}\right), 0 \leq i \leq I$, but $U_{i_{0}}\left(t_{0}\right)=0$ for a certain $i_{0} \in\{0, \ldots, I\}$. One can easily check that

$$
\begin{aligned}
& \frac{d U_{i_{0}}\left(t_{0}\right)}{d t}=\lim _{\epsilon \rightarrow 0} \frac{U_{i_{0}}\left(t_{0}\right)-U_{i_{0}}\left(t_{0}-\epsilon\right)}{\epsilon} \leq 0, \\
& \delta^{2} U_{i_{0}}\left(t_{0}\right)=\frac{U_{i_{0}+1}\left(t_{0}\right)-2 U_{i_{0}}\left(t_{0}\right)+U_{i_{0}-1}\left(t_{0}\right)}{h^{2}}>0 \quad \text { if } 1 \leq i_{0} \leq I-1, \\
& \delta^{2} U_{i_{0}}\left(t_{0}\right)=\frac{2 U_{1}\left(t_{0}\right)-2 U_{0}\left(t_{0}\right)}{h^{2}}>0 \quad \text { if } i_{0}=0, \\
& \delta^{2} U_{i_{0}}\left(t_{0}\right)=\frac{2 U_{I-1}\left(t_{0}\right)-2 U_{I}\left(t_{0}\right)}{h^{2}}>0 \quad \text { if } i_{0}=I,
\end{aligned}
$$

which implies that

$$
\begin{aligned}
& \frac{d U_{i_{0}}\left(t_{0}\right)}{d t}-\delta^{2} U_{i_{0}}\left(t_{0}\right)+\lambda U_{i_{0}}^{p}\left(t_{0}\right)<0, \quad \text { if } 0 \leq i_{0} \leq I-1, \\
& \frac{d U_{I}\left(t_{0}\right)}{d t}-\delta^{2} U_{I}\left(t_{0}\right)-\frac{2}{h} U_{I}^{q}\left(t_{0}\right)+\lambda U_{I}^{p}\left(t_{0}\right)<0, \quad \text { if } i_{0}=I .
\end{aligned}
$$

But these inequalities contradict (2)-(4) and we get the expected result.

Lemma 3 Let $U_{h}$ be a solution of (2)-(4) and the initial condition at (4) verifies

$$
\delta_{*}^{2} \varphi_{i}-\lambda \varphi_{i}^{p}>0, \quad 0 \leq i \leq I .
$$

Then, $\frac{d U_{i}(t)}{d t}>0$ for $0 \leq i \leq I, t \in\left[0, T_{h}\right)$.

Proof. Consider the functions $W_{i}(t)=\frac{d U_{i}(t)}{d t}, 0 \leq i \leq I, t \in\left[0, T_{h}\right)$. Let $t_{0}$ be the first $t \in\left(0, T_{h}\right)$ such that $W_{i}(t)>0$ for $t \in\left[0, t_{0}\right)$, but $W_{i_{0}}\left(t_{0}\right)=0$ for a certain $i_{0} \in\{0, \ldots, I\}$. We may assume without lost of generality that $i_{0}$ is the smallest integer which satisfies the above equality. Then we have

$$
\begin{aligned}
& \frac{d W_{i_{0}}\left(t_{0}\right)}{d t}=\lim _{k \rightarrow 0} \frac{W_{i_{0}}\left(t_{0}\right)-W_{i_{0}}\left(t_{0}-k\right)}{k} \leq 0, \quad 0 \leq i_{0} \leq I, \\
& \delta^{2} W_{i_{0}}\left(t_{0}\right)=\frac{W_{i_{0}+1}\left(t_{0}\right)-2 W_{i_{0}}\left(t_{0}\right)+W_{i_{0}-1}\left(t_{0}\right)}{h^{2}}>0, \quad 1 \leq i_{0} \leq I-1, \\
& \delta^{2} W_{i_{0}}\left(t_{0}\right)=\frac{2 W_{1}\left(t_{0}\right)-2 W_{0}\left(t_{0}\right)}{h^{2}}>0, \quad i_{0}=0, \\
& \delta^{2} W_{i_{0}}\left(t_{0}\right)=\frac{2 W_{I-1}\left(t_{0}\right)-2 W_{I}\left(t_{0}\right)}{h^{2}}>0, \quad i_{0}=I,
\end{aligned}
$$

which implies by a simple computation that

$$
\begin{array}{r}
\frac{d W_{i_{0}}\left(t_{0}\right)}{d t}-\delta^{2} W_{i_{0}}\left(t_{0}\right)+\lambda p U_{i_{0}}^{p-1}\left(t_{0}\right) W_{i_{0}}\left(t_{0}\right)<0 \quad \text { if } 0 \leq i_{0} \leq I-1, \\
\frac{d W_{I}\left(t_{0}\right)}{d t}-\delta^{2} W_{I}\left(t_{0}\right)+\left(\lambda p U_{I}^{p-1}\left(t_{0}\right)-\frac{2 q}{h} U_{I}^{p-1}\left(t_{0}\right)\right) W_{I}\left(t_{0}\right)<0 \quad \text { if } i_{0}=I .
\end{array}
$$

But inequalities (7)-(8) contradict (2)-(3), and the lemma is proved.

Lemma 4 Let $U_{h}$ be a solution of (2)-(4) and the initial condition at (4) verifies

$$
\varphi_{i}<\varphi_{i+1}, \quad 0 \leq i \leq I .
$$

Then, $U_{i}(t)<U_{i+1}(t), \quad 0 \leq i \leq I-1, t \in\left[0, T_{h}\right)$.

Proof. Let $t_{0}$ be the first $t>0$ such that $U_{i+1}(t)-U_{i}(t)>0$ for $t \in\left[0, t_{0}\right), 0 \leq i \leq I-1$, but $U_{i_{0}+1}(t)-U_{i_{0}}(t)=0$ for a certain $i_{0} \in\{0, \ldots, I-1\}$. We may suppose without lost of generality that $i_{0}$ is the smallest integer which verifies the 
above equality. Let us now consider the functions $Z_{i}(t)=U_{i+1}(t)-U_{i}(t)$ for $0 \leq i \leq I-1, t \in\left[0, T_{h}\right)$. We have

$$
\begin{aligned}
\frac{d Z_{i_{0}}\left(t_{0}\right)}{d t} & =\lim _{\epsilon \rightarrow 0} \frac{Z_{i_{0}}\left(t_{0}\right)-Z_{i_{0}}\left(t_{0}-\epsilon\right)}{\epsilon} \leq 0, \quad 0 \leq i_{0} \leq I-1, \\
\delta^{2} Z_{i_{0}}\left(t_{0}\right) & =\frac{Z_{i_{0}+1}\left(t_{0}\right)-2 Z_{i_{0}}\left(t_{0}\right)+Z_{i_{0}-1}\left(t_{0}\right)}{h^{2}}>0, \quad 1 \leq i_{0} \leq I-2, \\
\delta^{2} Z_{i_{0}}\left(t_{0}\right) & =\frac{Z_{1}\left(t_{0}\right)-3 Z_{0}\left(t_{0}\right)}{h^{2}}>0, \quad i_{0}=0, \\
\delta^{2} Z_{i_{0}}\left(t_{0}\right) & =\frac{Z_{I-2}\left(t_{0}\right)-3 Z_{I-1}\left(t_{0}\right)}{h^{2}}>0, \quad i_{0}=I-1,
\end{aligned}
$$

which implies that

$$
\begin{aligned}
& \frac{d Z_{i_{0}}\left(t_{0}\right)}{d t}-\delta^{2} Z_{i_{0}}\left(t_{0}\right)+\lambda\left(U_{i_{0}+1}^{p}\left(t_{0}\right)-U_{i_{0}}^{p}\left(t_{0}\right)\right)<0 \quad \text { if } 0 \leq i_{0} \leq I-2, \\
& \frac{d Z_{I-1}\left(t_{0}\right)}{d t}-\delta^{2} Z_{I-1}\left(t_{0}\right)-\frac{2}{h} U_{I}^{q}\left(t_{0}\right)+\lambda\left(U_{I}^{p}\left(t_{0}\right)-U_{I-1}^{p}\left(t_{0}\right)\right)<0 \text { if } i_{0}=I-1 .
\end{aligned}
$$

The inequalities (9)-(10) contradict (2)-(3) and the desired result follows.

The following theorem gives conditions under which the solution $U_{h}$ of (2)-(4) converges to the corresponding one of (1).

Theorem 1 Assume that the problem (1) has a solution $u \in C^{4,1}\left([0,1] \times\left[0, T_{d}\right]\right)$ and the initial condition at (4) verifies

$$
\left\|\varphi_{h}-u_{h}(0)\right\|_{\infty}=o(1) \text { as } h \rightarrow 0,
$$

where $u_{h}(t)=\left(u\left(x_{0}, t\right), \ldots, u\left(x_{I}, t\right)\right)^{T}$. Then, for h small enough, the semidiscrete problem (2)-(4) has a unique solution $U_{h} \in C^{1}\left(\left[0, T_{d}\right], \mathbb{R}^{I+1}\right)$ such that

$$
\max _{0 \leq t \leq T_{d}}\left\|U_{h}(t)-u_{h}(t)\right\|_{\infty}=O\left(\left\|\varphi_{h}-u_{h}(0)\right\|_{\infty}+h^{2}\right) \text { as } h \rightarrow 0 .
$$

Proof. Let $\gamma>0$ be such that

$$
\|u(\cdot, t)\|_{\infty} \leq \gamma \text { for } t \in\left[0, T_{d}\right] .
$$

Then the problem (2)-(4) has for each $h$, a unique solution $U_{h} \in C^{1}\left(\left[0, T_{h}\right), \mathbb{R}^{I+1}\right)$.

Let $t(h) \leq \min \left\{T_{d}, T_{h}\right\}$ be the greatest value of $t>0$ such that

$$
\left\|U_{h}(t)-u_{h}(t)\right\|_{\infty}<1 .
$$

The relation (11) implies $t(h)>0$ for $h$ small enough. Using the triangle inequality, we obtain

$$
\left\|U_{h}(t)\right\|_{\infty} \leq\|u(\cdot, t)\|_{\infty}+\left\|U_{h}(t)-u_{h}(t)\right\|_{\infty} \text { for } t \in(0, t(h)),
$$

which leads to

$$
\left\|U_{h}(t)\right\|_{\infty}<1+\gamma \text { for } t \in(0, t(h)) .
$$

Let $e_{h}(t)=U_{h}(t)-u_{h}(t), t \in(0, t(h))$ be the discretization error and consider the function

$$
Z(x, t)=\left(\left\|\varphi_{h}-u_{h}(0)\right\|_{\infty}+M h^{2}\right) e^{(L+1) t+C x^{2}}
$$

where $M, L, C$ are non-negative constants. We denote by $Z\left(x_{i}, t\right)$ the discretisation in space of $Z(x, t)$. For suitable non-negative constants $M, L, C$, we prove, using Lemma 1 that

$$
\left|e_{i}(t)\right|<Z\left(x_{i}, t\right), \quad 0 \leq i \leq I, t \in(0, t(h)), \quad \text { see (Taha et al., 2012) for more details. }
$$

We deduce that

$$
\left\|U_{h}(t)-u_{h}(t)\right\|_{\infty} \leq\left(\left\|\varphi_{h}-u_{h}(0)\right\|_{\infty}+M h^{2}\right) e^{(L+1) t+C}, \quad t \in(0, t(h)) .
$$

Now, we prove that $t(h)=T_{d}$. Suppose that $t(h)<T_{d}$. From (14) and (16), we obtain

$$
1=\left\|U_{h}(t(h))-u_{h}(t(h))\right\|_{\infty} \leq\left(\left\|\varphi_{h}-u_{h}(0)\right\|_{\infty}+M h^{2}\right) e^{(L+1) T_{d}+C} .
$$


Since the term on the right hand side of the above inequality goes to zero as $h$ tends to zero, we deduce that $1 \leq 0$, which is impossible.

\section{Numerical Blow-up}

From now on, we suppose $u_{0}(1) \geq 1$. Under suitable assumptions, we prove that the solution $U_{h}$ of the semidiscrete problem (2)-(4) blows up in finite time and that its semidiscrete blow-up time converges to the real one when the mesh size goes to zero.

We set

$$
\begin{array}{ll}
\left(H_{1}\right): & u_{0}>0, u_{0}^{\prime} \geq 0 \text { and } u_{0}^{\prime \prime}-\lambda u_{0}^{p}>0 \text { in }[0,1], \\
\left(H_{2}\right): & p \leq q \text { and } 0<\lambda<\frac{(q-1)(p+1)}{(q+1)(p-1)} .
\end{array}
$$

Theorem 2 Let $q>2$. Assume that the problem (1) has a solution $u$ which blows up in finite time $T$ such that $u \in$ $C^{4,1}([0,1] \times[0, T))$ and the initial condition at (4) verifies $\left\|\varphi_{h}-u_{h}(0)\right\|_{\infty}=o(1)$ as $h \rightarrow 0$. Under the assumptions $\left(H_{1}\right)$ and $\left(\mathrm{H}_{2}\right)$, the unique solution $U_{h}$ of (2)-(4) blows up in finite time $T_{h}$ for sufficiently small $h$, and we have :

$$
\lim _{h \rightarrow 0} T_{h}=T .
$$

Proof. For the proof, we use the Theorem 1.4 given in (Ushijima, 2000). We have to check conditions A0, A1'” and A2' of this theorem before applying it.

Step 1 (Condition A0) The solution $u$ of (1) blows up in finite time $T$ see (Gómez et al., 1993).

Step 2 (Condition A1"') We define the energy $I$ of problem (1) by

$$
I[u](t)=\frac{1}{2} \int_{0}^{1} u_{x}^{2}(x, t) d x-\frac{1}{q+1} u^{q+1}(1, t)+\frac{\lambda}{p+1} \int_{0}^{1} u^{p+1}(x, t) d x, \quad t \in[0, T) .
$$

For any solution $u$, this energy $I$ is monotone non-increasing function of $t$. In fact,

$$
\frac{d}{d t} I[u](t)=-\int_{0}^{1} u_{t}^{2}(x, t) d x \leq 0
$$

Because assumption $\left(H_{1}\right)$ holds, we know from (Gómez et al., 1993) that $u>0, u_{t} \geq 0$ and from (Chipot, Fila, \& Quittner, 1991) that $u_{x} \geq 0$.

Introduce the functional $J$ as follows :

$$
J[u](t)=\int_{0}^{1} u^{2}(x, t) d x, \quad t \in[0, T) .
$$

We have

$$
\begin{aligned}
\frac{d}{d t} J(t) & =2 \int_{0}^{1} u(x, t) u_{t}(x, t) d x \\
& =2\left(-2 I[u](t)+\frac{q-1}{q+1} u^{q+1}(1, t)-\lambda \frac{p-1}{p+1} \int_{0}^{1} u^{p+1}(x, t) d x\right) \\
& \geq-4 I[u](t)+2 \frac{(q-1)(p+1)-\lambda(q+1)(p-1)}{(q+1)(p+1)} u^{q+1}(1, t) \text { because } u_{0}(1) \geq 1 \\
& \geq-4 I\left[u_{0}\right]+2 \frac{(q-1)(p+1)-\lambda(q+1)(p-1)}{(q+1)(p+1)} u^{q+1}(1, t) \text { because } I \text { is non-increasing. }
\end{aligned}
$$

Set $\alpha=2 \frac{(q-1)(p+1)-\lambda(q+1)(p-1)}{(q+1)(p+1)}>0$ because of $H_{2}$.

Then we have

$$
\begin{aligned}
\frac{d}{d t} J(t) & \geq-4 I\left[u_{0}\right]+\alpha u^{q+1}(1, t) \\
& =-4 I\left[u_{0}\right]+\alpha\left(u^{q+1}(0, t)+(q+1) \int_{0}^{1} u_{x}(x, t) u^{q}(x, t) d x\right) .
\end{aligned}
$$


Since $u_{t}$ is nonnegative, we have $u_{x x} \geq 0$. Which implies that $u_{x}$ is a continuous and non-decreasing fonction with respect to $x$. Then, there exists $\xi(t) \in(0,1)$ such that $\int_{0}^{1} u_{x}(x, t) u^{q}(x, t) d x=u_{x}(\xi(t), t) \int_{0}^{1} u^{q}(x, t) d x$.

Denote $\Gamma_{1}=-4 I\left[u_{0}\right]+\alpha u_{0}^{q+1}(0)$ and $\Gamma_{2}=\inf \left\{\alpha(q+1) u_{x}(\xi(t), t), t \in[0, T]\right\}$.

Thus, we have

$$
\begin{aligned}
\frac{d}{d t} J(t) & \geq \Gamma_{1}+\Gamma_{2} \int_{0}^{1} u^{q}(x, t) d x \\
& \geq \Gamma_{1}+\Gamma_{2}(J)^{q / 2} .
\end{aligned}
$$

We obtain relation (21) by using Jensen's inequality.

Define $H(t)=-4 I\left[u_{0}\right]+\alpha u^{q+1}(1, t), t \in[0, T)$.

From (19), we have $\frac{d}{d t} J(t) \geq H(t), t \in[0, T)$ and $\lim _{t \rightarrow T} H(t)=\infty$ since the blow-up point of $u$ is $x=1$.

Now, for $t \in\left[0, T_{h}\right)$, we denote by

$$
\begin{gathered}
I_{h}\left[U_{h}\right](t)=\frac{1}{2 h} \sum_{i=0}^{I-1}\left(U_{i+1}(t)-U_{i}(t)\right)^{2}-\frac{1}{q+1} U_{I}^{q+1}(t)+\frac{\lambda h}{p+1} \sum_{i=0}^{I} U_{i}^{p+1}(t), \\
J_{h}(t)=h \sum_{i=0}^{I} U_{i}^{2}(t), \\
H_{h}(t)=-4 I\left[\varphi_{h}\right]+\alpha U_{I}^{q+1}(t),
\end{gathered}
$$

the numerical approximations of $I, J$ and $H$, respectively.

By a simple computation, we obtain for $t \in\left[0, T_{h}\right)$,

$$
\frac{d J_{h}(t)}{d t} \geq H_{h}(t) \text { and } \frac{d H_{h}(t)}{d t}=\alpha(q+1) U_{I}^{q}(t) \frac{d U_{I}(t)}{d t} \geq 0 .
$$

A straightforward calculation yields the following inequality

$$
\frac{d J_{h}(t)}{d t} \geq \Gamma_{1}^{\prime}+\Gamma_{2}^{\prime}\left(J_{h}\right)^{q / 2}
$$

where $\Gamma_{1}^{\prime}=-4 I\left[\varphi_{h}\right]+\alpha \varphi_{0}^{q+1}$ and

$\Gamma_{2}^{\prime}=\inf \left\{\alpha(q+1) \frac{U_{k_{0}+1}(t)-U_{k_{0}}(t)}{h}, t \in\left[0, T_{h}\right]\right\}>0$, with $k_{0} \in\{1, \ldots, I-2\}$ fixed.

Putting $G(s)=\Gamma_{1}^{\prime}+\Gamma_{2}^{\prime}(s)^{q / 2}$, it is clear that

$$
\frac{d J_{h}(t)}{d t} \geq G\left(J_{h}\right)
$$

and there exists $s_{0}>0$ such that

$$
\left\{\begin{array}{l}
G(s)>0 \text { for } s>s_{0}, \\
\int_{s_{0}}^{\infty} \frac{1}{G(s)} d s<\infty \text { since } q>2 .
\end{array}\right.
$$

Condition (A") of theorem 1.4 in (Ushijima, 2000) is satisfied.

Step 3 (Condition A2') By virtue of theorem 1, we show that for any $\epsilon>0$,

$$
\lim _{h \rightarrow 0} \sup _{t \in[0, T-\epsilon]}\left|J[u](t)-J_{h}\left[U_{h}\right](t)\right|=0 \text { and } \lim _{h \rightarrow 0} \sup _{t \in[0, T-\epsilon]}\left|H(t)-H_{h}(t)\right|=0 .
$$

Finally, conditions A0, A1"' and A2' are satisfied. According to theorem 1.4 of (Ushijima, 2000), we obtain the desired results. 


\section{Numerical Experiments}

In this section, we estimate the blow-up time of (2)-(4) by using the algorithm proposed by C. Hirota and K. Ozawa (Hirota \& Ozawa, 2006). This algorithm deals with the numerical blow-up time of ODEs (here, the semidiscrete scheme (2)-(4)). We first use the arc length transformation technique to transform the semidiscrete scheme (2)-(4) like this :

$$
\left\{\begin{array}{l}
\frac{d}{d \ell}\left(\begin{array}{c}
t \\
U_{0} \\
\vdots \\
U_{I}
\end{array}\right)=\frac{1}{\sqrt{1+\sum_{i=1}^{I} f_{i}^{2}}}\left(\begin{array}{c}
1 \\
f_{0} \\
\vdots \\
f_{I}
\end{array}\right), \quad 0<\ell<\infty, \\
t(0)=0, \quad U_{i}(0)=\varphi_{i}, \quad 0 \leq i \leq I,
\end{array}\right.
$$

where

$$
\begin{aligned}
f_{0} & =\frac{2}{h^{2}}\left(U_{1}-U_{0}\right)-\lambda U_{0}^{p}(t), \\
f_{i} & =\frac{1}{h^{2}}\left(U_{i+1}-2 U_{i}+U_{i-1}\right)-\lambda U_{i}^{p}(t), \quad 1 \leq i \leq I-1, \\
f_{I} & =\frac{2}{h^{2}}\left(U_{I-1}-U_{I}\right)+\frac{2}{h} U_{I}^{q}(t)-\lambda U_{I}^{p}(t),
\end{aligned}
$$

" ${ }^{\prime \prime}$ is such that $\ell^{2}=d t^{2}+\sum_{i=1}^{I} U_{i}^{2}$ and is called the arc length.

The variables $t$ and $U_{i}$ are fonctions of $\ell$, and it is proved in (Hirota \& Ozawa, 2006) that

$$
\lim _{\ell \rightarrow \infty} t(\ell)=T_{h} \quad \text { and } \quad \lim _{\ell \rightarrow \infty}\left\|U_{h}(\ell)\right\|_{\infty}=\infty .
$$

Then we introduce $\left\{\ell_{j}\right\}$, a sequence of the arc length and we apply an ODE solver to (25) for each value of $\left\{\ell_{j}\right\}$ in order to generate a sequence that converges linearly to the blow-up time. This sequence is finally accelerated by the Aitken $\Delta^{2}$ method see (Hirota \& Ozawa, 2006). As ODE solver, we have chosen DOP54. This code is MATLAB version of the well-known FORTRAN code DOPRI5 which has been written by Hairer and Wanner (Hairer, Nørsett, \& Wanner, 1993). We find in DOP54, three tolerances parameters, RelTol, AbsTol and InitialStep. RelTol and AbsTol parameters indicate the tolerances of relative and absolute errors respectively, and we use InitialStep to choose how errors are controlled, see (Hirota \& Ozawa, 2006) for more details. For our experiments we set RelTol= AbsTol $=1 . d-15$, InitialStep $=0$, $\ell_{j}=2^{10} \cdot 2^{j}(j=0, \ldots, 12)$ and the initial data

$$
\varphi_{i}=e^{i * h-\ln \left(e^{1}-1\right)}-\frac{i * h}{e^{1}-1}, \quad 0 \leq i \leq I .
$$

This initial data guarantees that if $q$ increases, the flow on the boundary also increases since $U_{I}(t) \geq \varphi_{I}=1, t>0$. But it can not ensure the growth of the absorption term in the equation by that of $p$ because $0<\varphi_{i} \leq 1, i=0, \ldots, I$. Obviously, if $\lambda$ increases, the absorption term in the equation also becomes large.

In the following tables, $T_{h}$ is the approximate blow-up time corresponding to meshes of $I=16,32,64,128,256,512,1024$, $n$ is the number of iterations and the order $(s)$ of the method is computed from

$$
s=\frac{\log \left(\left(T_{4 h}-T_{2 h}\right) /\left(T_{2 h}-T_{h}\right)\right)}{\log (2)}
$$

Table 1. For $p=2.5, q=2.5, \lambda=0.5$

\begin{tabular}{cccc}
\hline$I$ & $T_{h}$ & $n$ & $s$ \\
\hline 16 & 0.216071202 & 4154 & - \\
32 & 0.210906554 & 6952 & - \\
64 & 0.209320480 & 12404 & 1.70 \\
128 & 0.208851002 & 23101 & 1.76 \\
256 & 0.208715516 & 44733 & 1.79 \\
512 & 0.208677132 & 97408 & 1.82 \\
1024 & 0.208666410 & 293764 & 1.84
\end{tabular}

Table 2. For $p=2.5, q=3, \lambda=0.5$

\begin{tabular}{cccc}
\hline$I$ & $T_{h}$ & $n$ & $s$ \\
\hline 16 & 0.146361159 & 3086 & - \\
32 & 0.142014795 & 5194 & - \\
64 & 0.140656932 & 9289 & 1.68 \\
128 & 0.140250111 & 17290 & 1.74 \\
256 & 0.140131638 & 33341 & 1.78 \\
512 & 0.140097837 & 70399 & 1.81 \\
1024 & 0.140088342 & 202200 & 1.83 \\
\hline
\end{tabular}




\begin{tabular}{cccc}
\multicolumn{4}{c}{ Table 3. For $p=2.5, q=4, \lambda=0.5$} \\
\hline$I$ & $T_{h}$ & $n$ & $s$ \\
\hline 16 & 0.084607888 & 2020 & - \\
32 & 0.081038633 & 3425 & - \\
64 & 0.079895889 & 6155 & 1.64 \\
128 & 0.079548019 & 11473 & 1.72 \\
256 & 0.079445569 & 22048 & 1.76 \\
512 & 0.079416093 & 45078 & 1.80 \\
1024 & 0.079407758 & 120059 & 1.82 \\
\hline
\end{tabular}

\begin{tabular}{cccc}
\multicolumn{4}{c}{ Table 4 . For $p=2.5, q=3, \lambda=1$} \\
\hline$I$ & $T_{h}$ & $n$ & $s$ \\
\hline 16 & 0.184326256 & 3342 & - \\
32 & 0.178963408 & 5666 & - \\
64 & 0.177345272 & 10193 & 1.73 \\
128 & 0.176872688 & 19073 & 1.78 \\
256 & 0.176737698 & 37068 & 1.81 \\
512 & 0.176699759 & 81769 & 1.83 \\
1024 & 0.176689229 & 248435 & 1.85 \\
\hline
\end{tabular}

Table 5. For $p=2.5, q=3, \lambda=1.2$

\begin{tabular}{cccc}
\hline$I$ & $T_{h}$ & $n$ & $s$ \\
\hline 16 & 0.207781947 & 3481 & - \\
32 & 0.201749362 & 5923 & - \\
64 & 0.199961413 & 10684 & 1.75 \\
128 & 0.199446098 & 20047 & 1.79 \\
256 & 0.199300396 & 39162 & 1.82 \\
512 & 0.199259776 & 88792 & 1.84 \\
1024 & 0.199248575 & 276972 & 1.86 \\
\hline
\end{tabular}

\section{Remark 1}

- From the tables above, we can ensure the convergence of $T_{h}$ to the blow-up time of the solution of (1), since the order $(s)$ of the method goes to 2 , which is just the accuracy of the difference approximation in space.

- It comes from these tables that there is a relationship between the blow-up time, the flow on the boundary and the absorption term in the equation. In fact, when the absorption term in the equation is constant $(p=2.5, \lambda=0.5)$ and that the flow on the boundary increases (by $q=2.5$ to $q=4$ ), then the blow-up is accelerated (from 0.208 to 0.079 ) see Tables 1-3. Whereas when the flow on the boundary is a constant $(q=3)$ and that the absorption term in the equation becomes large (by $\lambda=0.5$ to 1.2 with $p=2.5$ ), then the blow-up is delayed (from 0.140 to 0.199 ) see Tables $2,4,5$.

- The numerical method used in this paper has some advantages. Firstly, to obtain the numerical blow-up, we are not obliged to put an additional condition on the initial data, which is not the case of some numerical methods (see Theorem 4.3, Theorem 8.6 in (Assalé et al., 2008) and Theorem 6 in (Adou et al., 2019)). Secondly, when the mesh size goes to zero, that is $I$ becomes large, with the method of this paper, the number of iterations $n$ increases slowly. This fact allow us to obtain easily results for high values of $I(I=512,1024)$.

Others illustrations are given by some plots in the below figures.

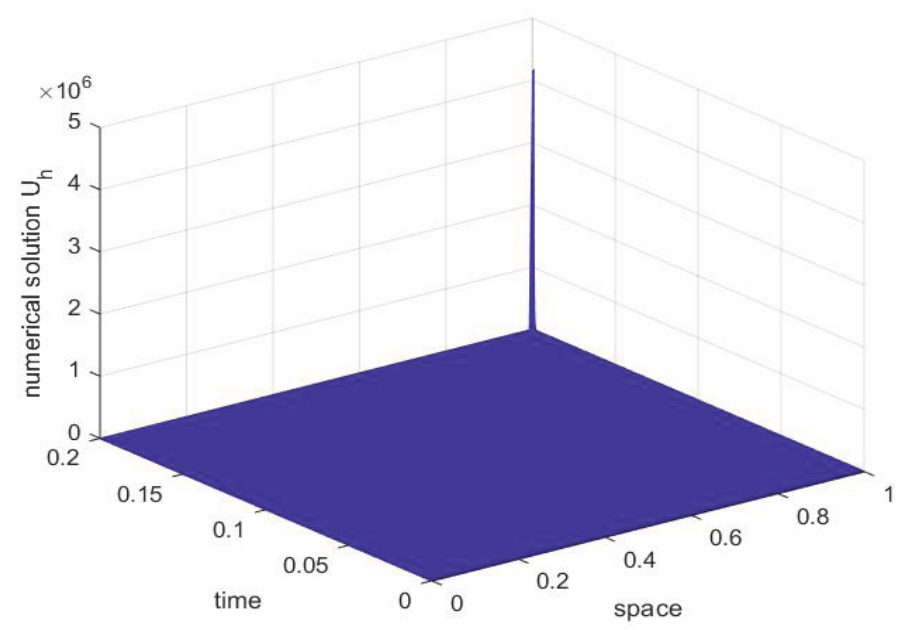

Figure 1. Evolution of the numerical solution for $I=128, p=2.5, q=3$ and $\lambda=1.2$ 


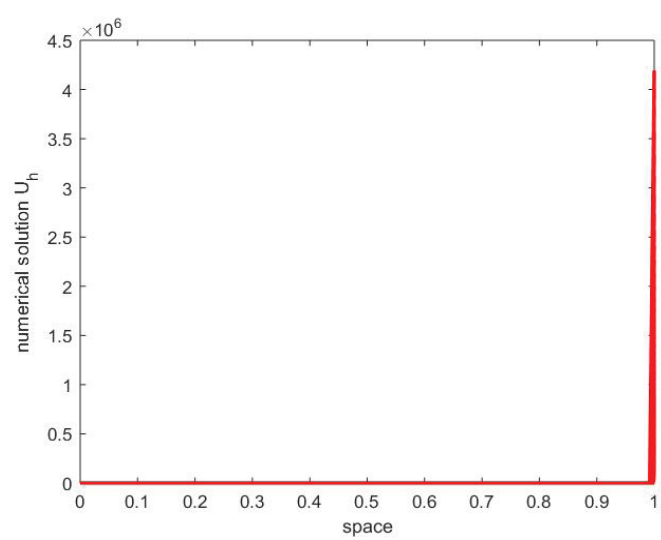

Figure 2. Evolution of $U_{h}$ according to the node for $I=128, p=2.5, q=3$ and $\lambda=1.2$

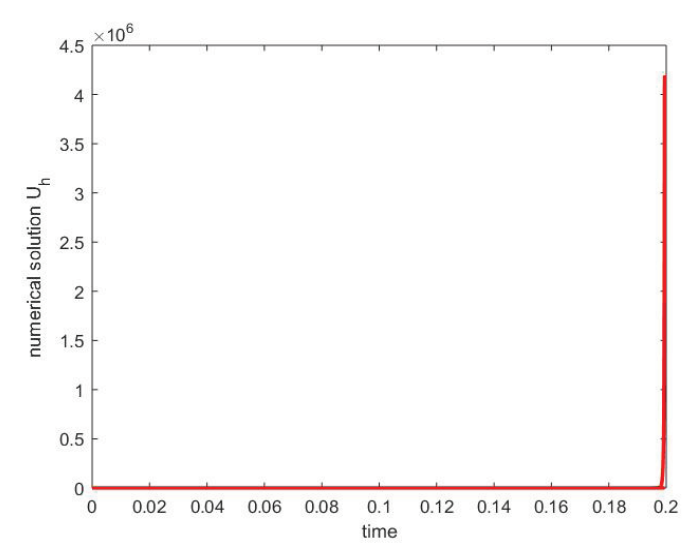

Figure 3. Evolution of $U_{h}$ according to the time for $I=128, p=2.5, q=3$ and $\lambda=1.2$

\section{Remark 2}

From the Figures 1, 2 and 3, we can observe that the numerical solution blows up in finite time at the last node, which is in agreement with the result established theoretically (Gómez et al., 1993 and Chipot et al., 1991).

\section{References}

Abia, L. M., López-Marcos, J. C., \& Martínez, J. (1996). Blow-up for semidiscretizations of reaction-diffusion equations. Appl. Numer. Math., 20, 145-156. https://doi.org/10.1016/0168-9274(95)00122-0

Adou, K. A., Touré, K. A., \& Coulibaly, A. (2019). On the computation of the numerical blow-up time for solutions of semilinear parabolic equation. International Journal of Numerical Methods and Applications, 18(1), 7-18. https://doi.org/10.17654/NM018010007

Assalé, L. A., Boni, T. K., \& Diabate, N. (2008). Numerical Blow-Up Time for a Semilinear Parabolic Equation with Nonlinear Boundary Conditions. Journal of Applied Mathematics, 2008, 1-30. https://doi.org/10.1155/2008/753518

Chipot, M., Fila, M., \& Quittner, P. (1991). Stationary solutions, blow up and convergence to stationary solutions for semilinear parabolic equations with nonlinear boundary conditions. Acta Math. Univ. Comenianae, LX(1), 35-103. https://doi.org/10.5167/uzh-22758

Dratman, E. (2010). Approximation of the solution of certain nonlinear ODEs with linear complexity. Journal of Computational and Applied Mathematics, 233, 2339-2350. https://doi.org/10.1016/j.cam.2009.10.019

Edja, K. B., Touré, K. A., \& Koua, B. J-C. (2018). Numerical Blow-up for A Heat Equation with Nonlinear Boundary Conditions. Journal of Mathematics Research, 10(5), 119-128. https://doi.org/10.5539/jmr.v10n5p119

Ganon, A., Taha, M. M., \& Touré, K. A. (2019). Numerical blow-up for a quasilinear parabolic equation with nonlinear boundary condition. Far East Journal of Mathematical Sciences (FJMS), 114(1), 19-38. https://doi.org/10.17654/MS114010019

Hairer, E., Nørsett, S. P., \& Wanner, G. (1993). Solving Ordinary Differential Equations I. Nonstiff problems. Springer Series in Comput. Math., 2nd ed., Springer-Verlag. https://doi.org/10.1007/978-3-540-78862-1

Hirota, C., \& Ozawa, K. (2006). Numerical method of estimating the blow-up time and rate of the solution of ordinary differential equations-An application to the blow-up problems of partial differential equations. Journal of Computational and Applied Mathematics, 193, 614-637. https://doi.org/10.1016/j.cam.2005.04.069

Gómez, J. L., Márquez, V., \& Wolanski, N. (1993). Dynamic behavior of positive solutions to reaction-diffusion problems with nonlinear absorption through the boundary. Rev. Unión Matemática Argentina, 38, 196-209. https://inmabb.criba.edu.ar/revuma/pdf/v38n3y4/p196-209.pdf

N'dri, K. C., Touré, K. A., \& Yoro, G. (2018). Numerical blow-up time for a parabolic equation with nonlinear boundary 
conditions. International Journal of Numerical Methods and Applications, 17, 141-160. https://doi.org/10.17654/NM017340141

Touré, K. A., N'Guessan, K., \& Diabate, N. (2015). Blow-up for Semidiscretizations of some Reaction-Diffusion Equations with a Nonlinear Convection Term. Global Journal of Pure and Applied Math, 11(6), 4273-4296.

Rossi, J. D. (1998). The Blow-up Rate for a Semilinear Parabolic Equation with a Nonlinear Boundary Condition. Acta Math. Univ. Comenianae, LXVII(2), 343-350.

Taha, M. M., Touré, K. A., \& Mensah, E. P. (2012). Numerical approximation of the blow-up time for a semilinear parabolic equation with nonlinear boundary conditions. Far East Journal of Mathematical Sciences (FJMS), 60(2), $125-167$.

Ushijima, T. K. (2000). On the Approximation of Blow-up Time for Solutions of Nonlinear Parabolic Equations. Publ. RIMS, Kyoto Univ., 36, 613-640. https://doi.org/10.2977/prims/1195142812

\section{Copyrights}

Copyright for this article is retained by the author(s), with first publication rights granted to the journal.

This is an open-access article distributed under the terms and conditions of the Creative Commons Attribution license (http://creativecommons.org/licenses/by/4.0/). 